\title{
КАЖУЩАЯСЯ ФОРМА НЕБЕСНОГО СВОДА
}

\section{Введение}

Среди многочисленных оптических иллюзий давно и хорошо известна кажущаяся сплюснутость небесного свода. Она проявляется главным образом в видимом увеличении диска солнца или луны и размеров созвездий при приближении этих светил к горизонту. До сих пор сколько-нибудь полная и убедительная теория этого эффекта отсутствует. В настоящей статье мы предложим основанную на простой идее количественную теорию, не только объясняющую сам факт сплюснутости небесного свода, но и позволяющую вывести уравнение, определяющее его форму. Будет показано, что небесный свод по своей кажущейся форме весьма близок к эллипсоиду.

\section{Исходные предпосылки}

Многие авторы (см., напр., обзор в [ $\left.{ }^{1}\right]$, с. $\left.202-215\right)$ указывают на недооценку горизонтальных расстояний как на причину рассматриваемой иллюзии. Вследствие этого возникает переоенка вертикальных углов, например, угла крутизны горного склона - склон кажется круче, чем на самом деле. Та же причина обусловливает и кажущуюся сплюснутость небесного свода. Если, например, направим взор под углом к горизонту, равным, как нам кажется, $45^{\circ}$, то, хотя истинный угол будет меньше, точка пересечения луча зрения с небесным сводом будет казаться делящей дугу между зенитом и горизонтом пополам. А так как нижняя из этих двух кажущихся равными половин соответствует на самом деле меньшему углу, а верхняя большему, то дуга, соединяющая зенит с горизонтом, должна представиться растянутой в горизонтальном направлении и сплюснутой в вертикальном.

В приведенном объяснении остается, однако, слабым местом исходное положение о недооценке горизонтальных расстояний. Оно принимается без достаточного обоснования. В нашей теории начнем именнว с количественного обоснования этого эффекта, причем изменим несколько постановку вопроса - вместо недооценки горизонтальных расстояний будем говорить о переоценке длин вертикальных линий.

Положим, нам нужно визуально оценить высоту какого-либо вертикального предмета (дерева, столба, стены здания и т. п.). Пусть оцениваемая высота достаточно велика по сравненію с размерами нашего тела (иначе высоту можно было бы оценить непосредственно, т. е. помимо оценки углов). Қак правило, оценка производится в этом слу- 


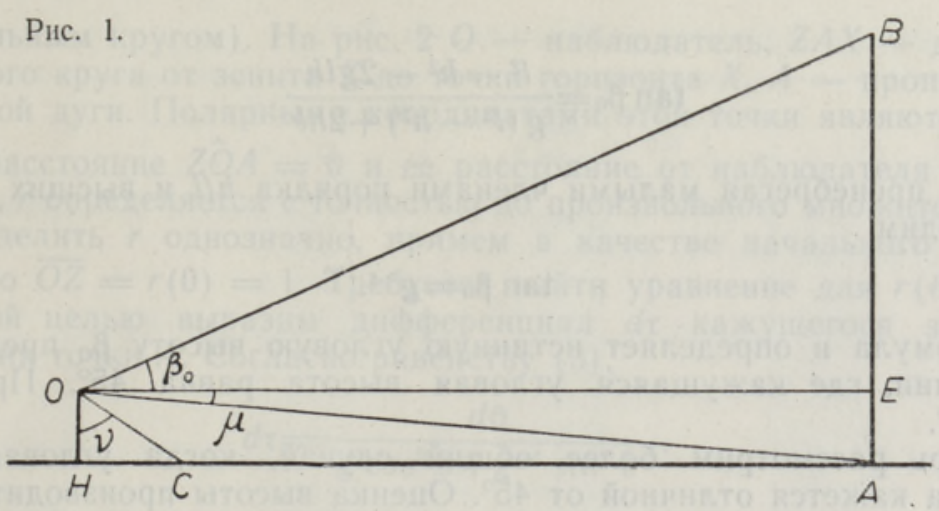

чае следующим образом. Мы приближаемся к предмету на расстояние, равное, как нам кажется, его высоте. О равенстве этих двух длин судим по равенству углов, под которыми они видны. Углы же равны тогда, когда высота предмета фактически меньше расстояния до него. Это обусловлено тем, что оба сравниваемых отрезка расположены относительно наблюдателя в различных направлениях. Но при оценке высоты это обстоятельство в силу каких-то психологических факторов не учитывается - равенство углов воспринимается как равенство длин. На рис. $1 A H-$ горизонтальная поверхность земли, $A B-$ предмет, $O H$ - наблюдатель, $O$ - его глаз. Пусть $\overline{H O}=h$. Наблюдатель считает высоту предмета равной его удаленности, если углы $B \hat{O} A$ и $A \hat{O} C$ равны, где $C$ - некоторая точка на поверхности земли перед наблюдателем, откуда он привык отсчитывать расстояния от себя вперед. Местоположение этой точки относительно наблюдателя определяется длиной $\overline{H C}=p$ и характеризуется отношением длин $p / h$, которое обозначим через g. Это число является, без сомнения, индивидуально обусловленным, но для каждого данного наблюдателя его можно считать постоянным. Оно всегда (или почти всегда) больше единицы. В этом убедимся впоследствии, когда окажется, что сплюснутость небесного свода имеет место именно при $g>1$, и притом в тем большей степени, чем $g$ больше.

Если $B \hat{O} A=A \hat{O} C$, то наблюдатель, считая высоту предмета равной его удаленности, оценивает его угловую высоту в $45^{\circ}$. Найдем истинную угловую высоту, принимая в качестве таковой угол $\beta_{0}=B \hat{O} E$, где $O E-$ горизонтальная линия (в силу условия $\overline{O H} \ll \overline{A B}$ этот угол практически равен $B \hat{H} A)$. Обозначим $\overline{A H}=l, A O E=\mu$ и $C \hat{O H}=v$. Тогда условие $B \hat{O A}=A \hat{O} C$ запишется в виде

откуда

$$
\beta_{0}+\mu=90^{\circ}-\mu-v,
$$

$$
\beta_{0}=90^{\circ}-2 \mu-v
$$

и

А так как

$$
\tan \beta_{0}=\operatorname{ctg}(2 \mu+v) .
$$

и

$$
\tan \mu=h / l
$$

$$
\tan v=g \text {, }
$$


To

$$
\tan \beta_{0}=\frac{l^{2}-h^{2}-2 g l h}{g\left(l^{2}-h^{2}\right)+2 h l} .
$$

Отсюда, пренебрегая малыми членами порядка $h / l$ и высших степеней $h / l$, находим

$$
\tan \beta_{0}=g^{-1}
$$

Эта формула и определяет истинную угловую высоту $\beta_{0}$ предмета на расстоянии, где кажущаяся угловая высота равна $45^{\circ}$. При $g>1$ $\beta_{0}<45^{\circ}$.

Теперь рассмотрим более общий случай, когда угловая высота предмета кажется отличной от $45^{\circ}$. Оценка высоты производится тогда опять путем сравнения высоты с удаленностью. Для этого наблюдатель находит на предмете (или его продолжении) точку, высота которой кажется ему равной удаленности предмета, а угловая высота равной $45^{\circ}$. Действительная угловая высота этой точки равна, как мы нашли, $\arctan g^{-1}$. Затем наблюдатель, сравнивая высоту предмета с высотой этой точки, находит, что высота предмета больше, скажем, в $k$ раз, и заключает, что тангенс угловой высоты равен $k$ (т. е. в $k$ раз больше $\tan 45^{\circ}$ ). А тангенс истинной угловой высоты равен, очевидно, $k \tan \beta_{0}=k g^{-1}$. Итак, обозначая истинную угловую высоту через $\beta$, а кажущуюся через $\psi$, находим

$$
\tan \psi=g \tan \beta .
$$

Равенство (1) является частным случаем этой формулы, когда $\psi=45^{\circ}$ и $\beta=\beta_{0}$.

\section{Основное уравнение}

В нижеследующем удобнее пользоваться вместо угловой высоты зенитным расстоянием. Обозначим истинное зенитное расстояние через $\vartheta$, а кажущееся через $\tau$, так что

$$
\tan \tau=g^{-1} \tan \vartheta \text {. }
$$

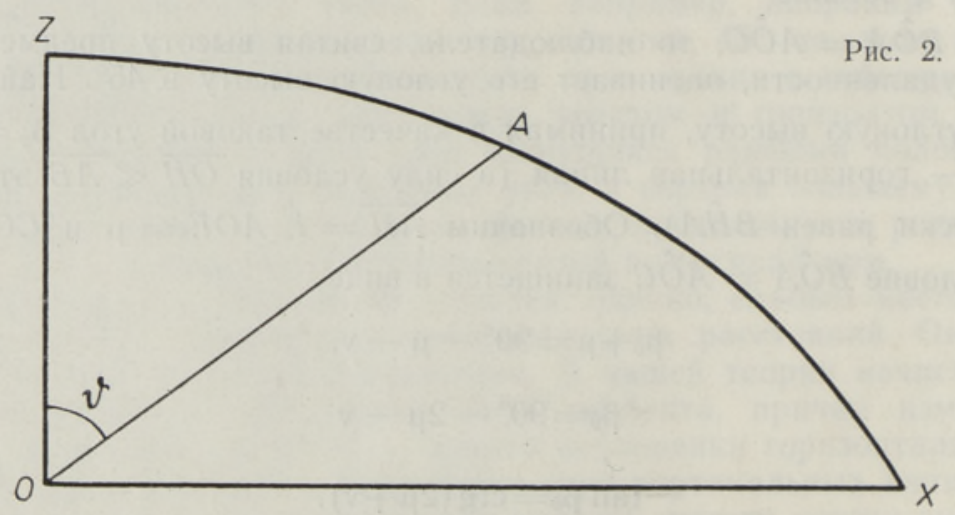

Выведем исходя из этой формулы дифференциальіос уравнение, определяющее кажущуюся форму линии пересечения небесного свода с вертикальной плоскостью (ниже будем условно называть эту линию 
вертикальным кругом). На рис. $2 O-$ наблюдатель, $Z A X-$ дуга вертикального круга от зенита $Z$ до точки горизонта $X, A-$ произвольная точка этой дуги. Полярными координатами этой точки являются ее зенитное расстояние $Z \hat{O} A=\vartheta$ и ее расстояние от наблюдателя $\overline{O A}=r$. Понятно, $r$ определяется с точностью до произвольного множителя. Чтобы определить $r$ однозначно, примем в качестве начального условия равенство $\overline{O Z}=r(0)=1$. Требуется найти уравнение для $r(\theta)$.

C этой целью выразим дифференциал $d \tau$ кажущегося зенитного расстояния точки $A$. Согласно равенству (3),

$$
d \tau=\frac{d \vartheta}{g \cos ^{2} \vartheta+g^{-1} \sin ^{2} \vartheta} .
$$

Но так как отсчитываемая от зенита длина дуги кажущегося вертикального круга пропорциональна кажущемуся зенитному расстоянию, то, обозначая дифференциал дуги через $d s$, имеем $d s=x d \tau$ или

$$
d s=\frac{x d \vartheta}{g \cos ^{2} \vartheta+g^{-1} \sin ^{2} \vartheta},
$$

где $x$ - зависящий от нормировки расстояний множитель пропорциональности. Отсюда получаем дифференциальное уравнение вертикального круга в виде

$$
\left(\frac{d r}{d \vartheta}\right)^{2}+r^{2}=\frac{x^{2}}{\left(g \cos ^{2} \vartheta+g^{-1} \sin ^{2} \vartheta\right)^{2}} .
$$

Чтобы определить здесь $x$, примем $\vartheta=0$. Тогда из условия $r(0)=1$ вместе с очевидным равенством $\left.\frac{d r}{d \vartheta}\right|_{0=0}=0$ получаем $x=g$. Итак, в окончательном виде дифференциальное уравнение вертикального круга таково:

$$
\left(\frac{d r}{d \vartheta}\right)^{2}+r^{2}=\left[1-\left(1-g^{-2}\right) \sin ^{2} \vartheta\right]^{-2} .
$$

Из этого уравнения, не решая его полностью, легко найти длину дуги $s(\vartheta)$ вертикального круга от зенита до произвольной точки. Именно, переписав уравнение в виде

$$
d s=\left[1-\left(1-g^{-2}\right) \sin ^{2} \vartheta\right]^{-1} d \vartheta=d\left(g \arctan \left(g^{-1} \tan \vartheta\right)\right)
$$

и интегрируя его, получаем

$$
s(\vartheta)=g \arctan \left(g^{-1} \tan \vartheta\right) .
$$

Переходим к решению уравнения (7).

\section{Решение основного уравнения}

Уравнение (7) не имеет решения в т. н. замкнутой форме, но можно найти решение в виде сходящегося ряда по степеням $\sin ^{2} \vartheta$. Положим

$$
r=1+\sum_{k=1}^{\infty} c_{k} \sin ^{2 k} \vartheta
$$


где $c_{k}$ - неопределенные коэффициенты. Тогда

$$
\frac{d r}{d \vartheta}=\sum_{k=1}^{\infty} 2 k c_{k} \sin ^{2 k-1} \vartheta \cos \vartheta
$$

Подставляя (10) и (11) в уравнение (7) и разлагая его правую часть тоже по степеням $\sin ^{2} \vartheta$, находим

$$
\begin{gathered}
2 \sum_{k=1}^{\infty} c_{k} \sin ^{2 k} \vartheta-\sum_{k=1}^{\infty} \sum_{l=1}^{\infty}(4 k l-1) c_{k} c_{l} \sin ^{2 k+2 l} \vartheta+ \\
+4 \sum_{k=1}^{\infty} \sum_{l=1}^{\infty} k l c_{k} c_{l} \sin ^{2 k+2 l-2} \vartheta=\sum_{k=1}^{\infty}(k+1)\left(1-g^{-2}\right)^{k} \sin ^{2 k} \vartheta .
\end{gathered}
$$

Отсюда, приравнивая коэффициенты при одинаковых степенях $\sin ^{2} \theta$ справа и слева, находим последовательность рекуррентных уравнений, определяющих все коэффициенты $c_{k}$. Для $c_{1}$ получаем квадратное уравнение

$$
4 c_{1}^{2}+2 c_{1}-2\left(1-g^{-2}\right)=0,
$$

откуда

$$
c_{1}=\frac{-g+\sqrt{9 g^{2}-8}}{4 g} .
$$

Остальные коэффициенты определяются из линейных уравнений, общий вид которых таков:

$$
\begin{gathered}
2 c_{k}-(k+1)\left(1-g^{-2}\right)^{k}+4 \sum_{l=0}^{k-1}(l+1)(k-l) c_{l+1} c_{k-l}- \\
-\sum_{l=1}^{k-1}[4 l(k-l)-1] c_{l} c_{k-l}=0, \quad k=2,3, \ldots
\end{gathered}
$$

Отсюда

$c_{k}=\frac{(k+1)\left(1-g^{-2}\right)^{k}+\sum_{l=1}^{k-1}[4 l(k-l)-1] c_{l} c_{k-l}-4 \sum_{l=1}^{k-2}(l+1)(k-l) c_{l+1} c_{k-l}}{2\left(1+4 k c_{1}\right)}$,

$$
k>1 \text {. }
$$

В табл. 1 приведены значения коэффициентов $c_{k}$ до $k=15$ при $g$, равном, для примера, $1,25,1,50$ и 1,75 .

Сходимость ряда (10) вытекает из сходимости ряда в правой части уравнения (12). При не слишком близких к единице значениях аргумента ряд сходится достаточно быстро, но с ростом $\sin \vartheta$ сходимость ухудшается настолько, что ряд становится практически мало пригодным. Поэтому приведем вдобавок способ численного решения уравнения, эффективный во всем интервале $0 \leqslant \sin \vartheta \leqslant 1$.

Пусть (см. рис. 3) кривая $Z A_{1} A_{2} X$ изображает искомое решение, а $A_{1}, A_{2}$ - две точки на ней, соответствующие близким значениям зенитного расстояния $\vartheta_{1}$ и $\vartheta_{2}$. Обозначим $\overline{O A}_{1}=r\left(\vartheta_{1}\right)=r_{1}, \overline{O A_{2}}=$ $=r\left(\vartheta_{2}\right)=r_{2}$ и $\overline{A_{1} A_{2}}=q$. Пусть в последовательном вычислении значений радиальной координаты $r_{1}$ уже найдено и требуется вычислить $r_{2}$. Для этого из треугольника $O A_{1} A_{2}$ имеем 
Таблица 1

\begin{tabular}{r|c|c|c}
\hline$k$ & \multicolumn{3}{c}{$c_{k}$} \\
\cline { 2 - 4 } & $g=1,25$ & $g=1,50$ & $g=1,75$ \\
\hline 1 & 0,24244 & 0,33333 & 0,38185 \\
2 & 0,09613 & 0,17172 & 0,22173 \\
3 & 0,04669 & 0,10154 & 0,14515 \\
4 & 0,02625 & 0,06523 & 0,10131 \\
5 & 0,01656 & 0,04460 & 0,07388 \\
6 & 0,01141 & 0,03209 & 0,05574 \\
7 & 0,00839 & 0,02409 & 0,04326 \\
8 & 0,00648 & 0,01876 & 0,03441 \\
9 & 0,00519 & 0,01505 & 0,02796 \\
10 & 0,00427 & 0,01239 & 0,02316 \\
11 & 0,00360 & 0,01041 & 0,01950 \\
12 & 0,00308 & 0,00891 & 0,01667 \\
13 & 0,00267 & 0,00773 & 0,01443 \\
14 & 0,00235 & 0,00679 & 0,01264 \\
15 & 0,00208 & 0,00603 & 0,01119
\end{tabular}

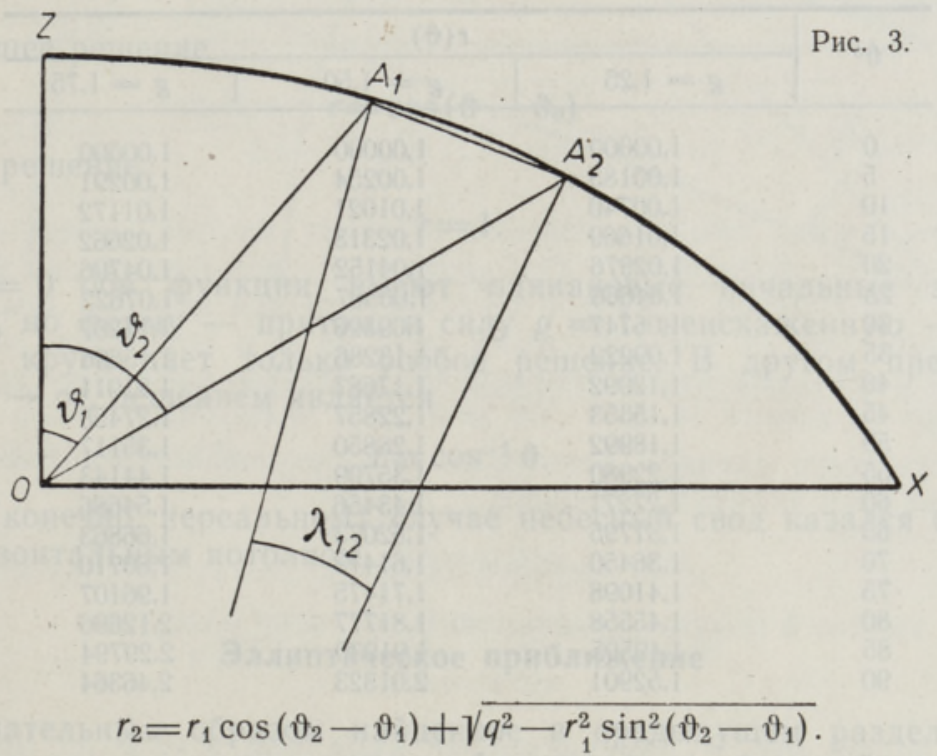

Применение этой формулы предполагает знание длины хорды $q$. В первом приближении можно заменить $q$ длиной дуги $s\left(\vartheta_{2}\right)-s\left(\vartheta_{1}\right)$, равной, согласно формуле (9),

$$
s_{2}-s_{1}=g\left[\arctan \left(g^{-1} \tan \vartheta_{2}\right)-\arctan \left(g^{-1} \tan \vartheta_{1}\right)\right]
$$

(здесь $s_{2}=s\left(\vartheta_{2}\right)$ и $\left.s_{1}=s\left(\vartheta_{1}\right)\right)$. Итак, подставив вместо $q$ в формулу (16) $s_{2}-s_{1}$, найдем приближенное значение $r_{2}$.

Если шаг $\vartheta_{2}-\vartheta_{1}$ достаточно мал, то этим приближением можно ограничиться. Если же $\vartheta_{2}-\vartheta_{1}$ не столь мало или если хотим получить бо́льшую точность, то нужно перейти к следующему приближению. Для этого проведем в точках $A_{1}$ и $A_{2}$ нормали к кривой (см. рис. 3). Угол $\lambda_{12}$, образуемый нормалями, равен, очевидно,

$$
\lambda_{12}=\vartheta_{2}-\vartheta_{1}-\arctan \left(\frac{d r}{r d \vartheta}\right)_{2}+\arctan \left(\frac{d r}{r d \theta}\right)_{1}
$$

или, согласно уравнению (7), 


$$
\begin{gathered}
\lambda_{12}=\vartheta_{2}-\vartheta_{1}-\arccos \left\{r_{2}\left[1-\left(1-g^{-2}\right) \sin ^{2} \vartheta_{2}\right]\right\}+ \\
+\arccos \left\{r_{1}\left[1-\left(1-g^{-2}\right) \sin ^{2} \vartheta_{1}\right]\right\} .
\end{gathered}
$$

Если заменим дугу $s_{2}-s_{1}$ приближенно дугой окружности, то радиус ее будет равен $\left(s_{2}-s_{1}\right) \lambda_{12}{ }^{-1}$, а длина $q$ хорды $A_{1} A_{2}$ выразится как

$$
q=\left(s_{2}-s_{1}\right) \cdot \frac{\sin \left(\lambda_{12} / 2\right)}{\lambda_{12} / 2} \text {. }
$$

Здесь $\frac{\sin \left(\lambda_{12} / 2\right)}{\lambda_{12} / 2}$ есть поправочный множитель к формуле $q=s_{2}-s_{1}$, использованной выше в первом приближении. Он, конечно, мало отличается от единицы. Вычисление его по формуле (18) предполагает знание $r_{2}$, но там можно взять значение этой величины, полученное в первом приближении. А второе приближение получим подстановкой в

\begin{tabular}{|c|c|c|c|}
\hline \multirow{2}{*}{$\theta^{0}$} & \multicolumn{3}{|c|}{$r(\theta)$} \\
\hline & $g=1,25$ & $g=1,50$ & $g=1,75$ \\
\hline 0 & 1,00000 & 1,00000 & . 1,00000 \\
\hline 5 & 1,00185 & 1,00254 & 1,00291 \\
\hline 10 & 1,00740 & 1,01021 & 1,01172 \\
\hline 15 & 1,01669 & 1,02313 & 1,02662 \\
\hline 20 & 1,02976 & 1,04152 & 1,04796 \\
\hline 25 & 1,04666 & 1,06567 & 1,07622 \\
\hline 30 & 1,06747 & 1,09596 & 1,11207 \\
\hline 35 & 1,09222 & 1,13286 & 1,15636 \\
\hline 40 & 1,12092 & 1,17687 & 1,21011 \\
\hline 45 & 1,15353 & 1,22857 & 1,27458 \\
\hline 50 & 1,18992 & 1,28850 & 1,35117 \\
\hline 55 & 1,22980 & 1,35709 & 1,44143 \\
\hline 60 & 1,27271 & 1,43456 & 1,54686 \\
\hline 65 & 1,31795 & 1,52073 & 1,66863 \\
\hline 70 & 1,36450 & 1,61473 & 1,80710 \\
\hline 75 & 1,41098 & 1,71475 & 1,96107 \\
\hline 80 & 1,45558 & 1,81777 & 2,12696 \\
\hline 85 & 1,49595 & 1,91930 & 2,29794 \\
\hline 90 & 1,52901 & 2,01323 & 2,46364 \\
\hline
\end{tabular}
формулу (16) значения $q$ из формулы (19).

Таблица 2

Как показывает практический расчет, этот метод дает при $\vartheta_{2}-\vartheta_{1}=5^{\circ}$ значения $r$ с относительной ошибкой менее $10^{-5}$. Принимая опять $g$ равным $1,25,1,50$ и 1,75 , мы табулировали функцию $r(\vartheta)$ (см. табл. 2). В части таблицы (до $\vartheta=55^{\circ}$ ) вычисления выполнены обоими методами (т. е. с помощью ряда (10) и вышеизложенным численным методом) и дали совпадающие до пятого знака̇ результаты. В остальной части таблицы, где ряд (10) сходится плохо, применен только численный метод. На рис. 4 показан вид четверти вертикального круга для тех же значений $g$.

Сделаем еще одно замечание. В формуле (13) мы выбрали перед корнем знак плюс. Выбрав минус, мы получили бы другое решенне. В численном методе, аналогично, другому решению соответствует обратный знак корня в формуле (16). Эти два решения удовлетворяют одному и тому же начальному условию $r(0)=1$, но отличаются друг от друга знаком пронзводной $d r / d \vartheta$. В выбранном нами решении $d r / d \vartheta>0$, как и должно быть, если небесный свод сплюснут. Другое решение никакого отношения $\mathrm{K}$ видимой форме небесного свода не 


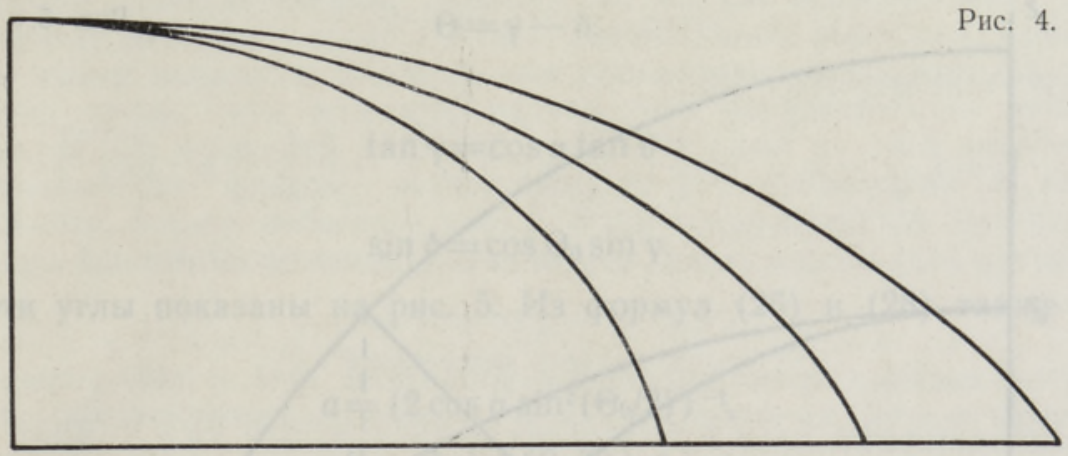

имеет. В предельном случае $g=1$ одно из решений является особым, другое - частным. В самом деле, уравнение

$$
\left(\frac{d r}{d \vartheta}\right)^{2}+r^{2}=1
$$

имеет общее решение

$$
r=\cos \left(\vartheta-\vartheta_{0}\right)
$$

и особое решение

$$
r=1 \text {. }
$$

При $\vartheta_{0}=0$ обе функции имеют одинаковые начальные значения $r(0)=1$, но форму - притом, в силу $g=1$, неискаженную - вертикального круга дает только особое решение. В другом предельном случае, $g \rightarrow \infty$, решением является

$$
r=\cos ^{-1} \vartheta \text {. }
$$

В этом (конечно, нереальном) случае небесный свод казался бы плоским горизонтальным потолком.

\section{Эллиптическое приближение}

Замечательным образом найденное в предыдущем разделе решение основного уравнения аппроксимируется с большой точностью эллипсом, центр которого лежит на вертикали ниже земной поверхности. Обозначим полуоси эллипса через a (горизонтальная, большая) и $b$ (вертикальная, малая). Чтобы эллипс проходил через зенит на единичном расстоянии от наблюдателя, его центр должен лежать на $b-1$ ниже земной поверхности. На рис. $5 O-$ наблюдатель, $O X-$ горизонталь, $Z$ - зенит, $C$ - центр эллипса, $Z P X$ - дуга эллипса от зенита до горизонта. В декартовых осях $x z$ уравнение эллипса имеет вид

$$
a^{-2} x^{2}+b^{-2}(z+b-1)^{2}=1,
$$

а в полярных координатах $\varrho$, $\vartheta$ оно таково:

$$
\varrho(\vartheta)=\frac{-a^{2}(b-1) \cos \vartheta+a b \sqrt{a^{2} \cos ^{2} \vartheta+(2 b-1) \sin ^{2} \vartheta}}{a^{2} \cos ^{2} \vartheta+b^{2} \sin ^{2} \vartheta} .
$$

Нам предстоит убедиться в том, что это выражение, при надлежащем 


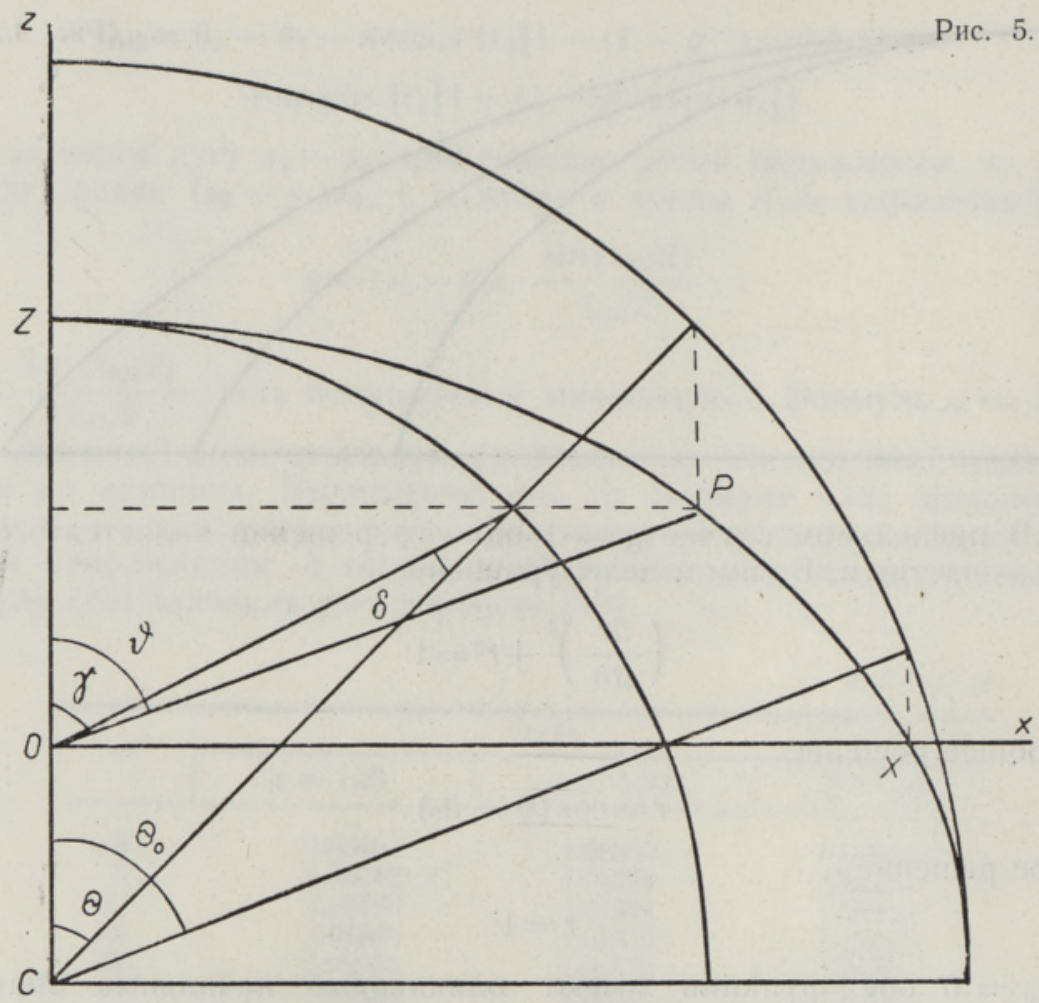

выборе $a$ и $b$, совпадает с большой точностью с решением (10) уравнения (7).

Прежде всего сделаем некоторые преобразования и выразим дифференциал дуги эллипса. Положим

$$
\cos \alpha=b / a
$$

и введем вместо $\vartheta$ новую переменную $\Theta$ согласно равенству

$$
\sin \Theta=x / a \text {. }
$$

Отсюда видно, что $\Theta$ изменяется от нуля при $\vartheta=0$ до некоторого значения $\Theta_{0}$ при $\vartheta=90^{\circ}$, причем $\Theta_{0}$ определяется из уравнения (24) формулой

$$
\cos \Theta_{0}=1-b^{-1} .
$$

Отсюда и из того же уравнения (24) следует

$$
z / b=\cos \Theta-\cos \Theta_{0} \text {. }
$$

Следовательно,

$$
\tan \vartheta=x / z=(\sin \Theta / \cos \alpha)\left(\cos \Theta-\cos \Theta_{0}\right)^{-1}
$$

и

$$
\varrho=\left(x^{2}+z^{2}\right)^{1 / 2}=a \sqrt{\sin ^{2} \Theta+\cos ^{2} \alpha\left(\cos \Theta-\cos \Theta_{0}\right)^{2}} .
$$

Формулу (30) можно обратить: 


$$
\Theta=\gamma-\delta
$$

где

$$
\tan \gamma=\cos \alpha \tan \vartheta
$$

и

$$
\sin \delta=\cos \Theta_{0} \sin \gamma .
$$

Все эти углы показаны на рис. 5. Из формул (26) и (28) также следует

$$
\begin{gathered}
a=\left(2 \cos \alpha \sin ^{2}\left(\Theta_{0} / 2\right)\right)^{-1}, \\
b=\left(2 \sin ^{2}\left(\Theta_{0} / 2\right)\right)^{-1} .
\end{gathered}
$$

Дифференциал дуги эллипса выражается как

$$
d \sigma=a \sqrt{1-\sin ^{2} \alpha \sin ^{2} \Theta} d \Theta .
$$

Перепишем еще формулу (25), выражая, согласно формулам (35) и (36), $a$ и $b$ через $\alpha$ и $\Theta_{0}$ :

$$
\varrho(\vartheta)=\frac{-\cos \Theta_{0} \cos \vartheta+\sqrt{1-\sin ^{2} \vartheta\left(1-\sin ^{2} \Theta_{0} \cos ^{2} \alpha\right)}}{2 \sin ^{2}\left(\Theta_{0} / 2\right)\left(1-\sin ^{2} \alpha \sin ^{2} \vartheta\right)} .
$$

Теперь сформулируем условия, обеспечивающие близость эллипса к кривой, определяемой решением (10) уравнения (7). Условий должно быть два, так как определению подлежат полуоси $a, b$ эллипса или, иначе, величины $\alpha$ и $\Theta_{0}$ (см. формулы (26) и (28) или (35) и (36)). В качестве первого условия примем равенство длин дуг обеих кривых в полном интервале $0^{\circ} \leqslant \vartheta \leqslant 90^{\circ}$, т. е. от зенита до горнзонта. Согласно равенству (9)

$$
s\left(90^{\circ}\right)=g \pi / 2 .
$$

Длина дуги эллипса равна, согласно формуле (37),

$$
\sigma\left(90^{\circ}\right)=a \int_{0}^{\Theta_{0}} \sqrt{1-\sin ^{2} \alpha \sin ^{2} \Theta} d \Theta
$$

T. e.

$$
\sigma\left(90^{\circ}\right)=a E\left(\alpha, \Theta_{0}\right),
$$

где $E\left(\alpha, \Theta_{0}\right)$ - эллиптический интеграл 2 -го рода с аргументом $\Theta_{0}$ и параметром $\alpha$. Итак, потребуем, чтобы

$$
E\left(\alpha, \Theta_{0}\right)=g \pi \cos \alpha \sin ^{2}\left(\Theta_{0} / 2\right)
$$

(здесь учтена формула (35)).

Второе услювие получим следующим образом. Решая уравнение (7), мы разложили $r(\vartheta)$ в ряд. Аналогично можем разложить в ряд $\varrho(\vartheta)$ из формулы (38). Чтобы обе функции были возможно близки друг к другу, потребуем, чтобы коэффициенты при $\sin ^{2} \vartheta$ были в обонх разложениях одинаковы. Из формулы (38) находим

$$
\varrho=1+\left[\frac{1}{2}-\cos ^{2} \alpha \sin ^{2}\left(\Theta_{0} / 2\right)\right] \sin ^{2} \vartheta+\ldots
$$

Отсюда получаем второе условие в виде 


$$
\cos \alpha \sin \left(\Theta_{0} / 2\right)=C,
$$

где, согласно формуле (13),

$$
C=\left(\frac{1}{2}-c_{1}\right)^{1 / 2}=\frac{1}{2}\left[\left(\frac{3}{2}+g^{-1} \sqrt{2}\right)^{1 / 2}-\left(\frac{3}{2}-g^{-1} \sqrt{2}\right)^{1 / 2}\right] .
$$

Равенство (42) можем теперь переписать в более простом виде

$$
E\left(\alpha, \Theta_{0}\right)=g \pi C \sin \left(\Theta_{0} / 2\right) .
$$

Итак, система трансцендентных уравнений (44) и (46) определяет величины $\alpha$ и $\Theta_{0}$, из которых в свою очередь по формулам (35) и (36) вычисляются полуоси $a$ и $b$.

Перейдем к оценке точности эллиптического приближения. Для этого сравним, во-первых, длины дуг вертикального круга - точную $s(\theta)$ : приближенную $\sigma(\vartheta)$, и, во-вторых, радиальные координаты - точную $r(\vartheta)$ и приближенную $\varrho(\vartheta)$. Для $s(\vartheta)$ уже имеем формулу (9). Формулу для $\sigma(\vartheta)$ получим, интегрируя дифференциал (37). С учетом формул (35) и (44) находим

$$
\sigma(\vartheta)=\left[2 C \sin \left(\Theta_{0} / 2\right)\right]^{-1} E(\alpha, \Theta),
$$

где $\Theta$ выражается через $\vartheta$ с помощью формул (32)-(34). Из формул (9) и (47) следует

$$
s(\vartheta)-\sigma(\vartheta)=g \arctan \left(g^{-1} \tan \vartheta\right)-\left[2 C \sin \left(\Theta_{0} / 2\right)\right]^{-1} E(\alpha, \Theta) .
$$

Далее, разлагая $\varrho(\vartheta)$ из формулы (38) в ряд и пользуясь разложением (10), находим

$$
r(\vartheta)-\varrho(\vartheta)=\sum_{k=0}^{\infty} d_{k} \sin ^{2 k+4} \vartheta
$$

где

$$
d_{k}=c_{k+2}-c_{1} \sin ^{2 h+2} \alpha-
$$

$-\sin ^{-2}\left(\Theta_{0} / 2\right) \sum_{l=0}^{k} \frac{(2 l+1) ! \sin ^{2 k-2 l} \alpha\left[\cos \Theta_{0}-\left(1-\sin ^{2} \Theta_{0} \cos ^{2} \alpha\right)^{l+2}\right]}{2^{2 l+3 l !}(l+2) !}$.

Из последней формулы видно, что коэффициенты $d_{k}$ можно вычислять по рекуррентной формуле

$$
\begin{gathered}
d_{k+1}=c_{k+3}+\left(d_{k}-c_{k+2}\right) \sin ^{2} \alpha- \\
-\frac{(2 k+3) !}{2^{2 k+5}(k+1) !(k+3) !} \cdot \frac{\cos \Theta_{0}-\left(1-\sin ^{2} \Theta_{0} \cos ^{2} \alpha\right)^{k+3}}{\sin ^{2}\left(\Theta_{0} / 2\right)},
\end{gathered}
$$

причем

$$
\begin{aligned}
d_{0}= & c_{2}-c_{1} \sin ^{2} \alpha-\frac{1}{2} \cos ^{2} \alpha \cos ^{2}\left(\Theta_{0} / 2\right)+ \\
& +\frac{1}{4} \cos ^{4} \alpha \sin ^{2} \Theta_{0} \cos ^{2}\left(\Theta_{0} / 2\right)+\frac{1}{8} .
\end{aligned}
$$

Как увидим в следующем разделе, коэффициенты $d_{k}$ фактически очень малы. Поэтому и отличие $\varrho(\vartheta)$ от $r(\vartheta)$ очень мало. Это подтверждается и непосредственным сравнением обеих величин. Разность $s(\vartheta)-\sigma(\vartheta)$ тоже очень мала. Поскольку речь идет о субъективном явлении, не поддающемся точному измерению, то эти расхождения 
практически несущественны. Поэтому мы вправе отождествлять видимую форму небесного свода с эллипсоидом. Наши выкладки, приводящие к этому выводу, с точки зрения рассматриваемого эффекта даже излишне точны; нам кажется, однако, что аппроксимация решения уравнения (7) функцией вида (38) является сама по себе интересным математическим фактом, независимо от его применения в теории кажущейся формы небесного свода. Этим оправдывается чрезмерная, казалось бы, точность наших расчетов.

\section{Численные результаты}

В табл. 2 уже был приведен численный расчет формы вертикального круга для трех значений g. В этом разделе дополним эти данные

Таблица 3

Таблица 4

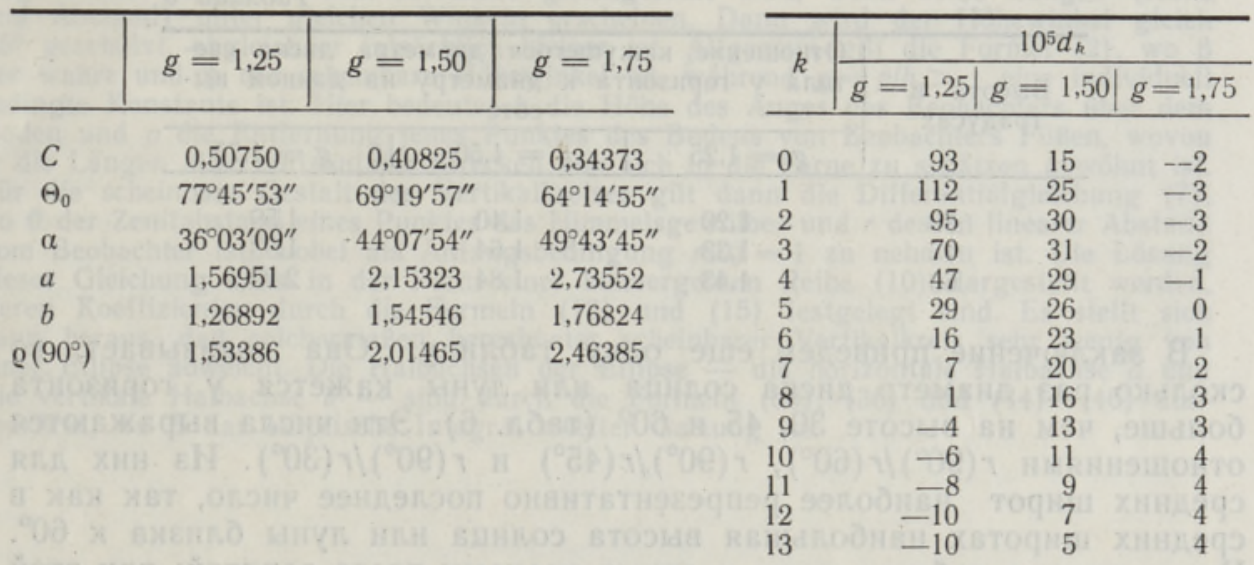

Таблища 5

\begin{tabular}{l|c|c|c}
\hline \multirow{2}{*}{$0^{0}$} & $g=1,25$ & $g=1,50$ & $g=1,75$ \\
\cline { 2 - 4 } & $10^{5}(r-\varrho) \mid 10^{5}(s-\sigma)$ & $10^{5}(r-\varrho)\left|10^{5}(s-\sigma)\right| 10^{5}(r-\varrho) \mid 10^{5}(s-\sigma)$ \\
\hline
\end{tabular}

$\begin{array}{rrrrrrr}0 & 0 & 0 & 0 & 0 & 0 & 0 \\ 5 & 0 & 0 & 0 & 0 & 0 & 0 \\ 10 & 0 & 0 & 0 & 0 & 0 & 0 \\ 15 & 1 & 0 & 0 & 0 & 0 & 0 \\ 20 & 2 & 0 & 0 & 0 & 0 & 0 \\ 25 & 3 & 1 & 1 & 0 & 0 & 0 \\ 30 & 8 & 2 & 1 & 1 & 0 & 0 \\ 35 & 16 & 7 & 3 & 2 & 0 & 0 \\ 40 & 28 & 13 & 5 & 3 & -1 & 0 \\ 45 & 46 & 21 & 10 & 6 & -1 & 0 \\ 50 & 74 & 33 & 19 & 11 & -2 & 0 \\ 55 & 122 & 55 & 31 & 20 & -3 & 0 \\ 60 & 160 & 85 & 50 & 33 & -4 & 1 \\ 65 & 216 & 119 & 80 & 55 & -3 & 2 \\ 70 & 269 & 160 & 119 & 85 & 2 & 3 \\ 75 & 297 & 192 & 161 & 118 & 8 & 10 \\ 80 & 252 & 202 & 187 & 145 & 32 & 23 \\ 85 & 46 & 152 & 134 & 131 & 47 & 37 \\ 90 & -485 & 0 & -142 & 0 & -21 & 0\end{array}$

результатами, относящимися к эллиптическому приближению (при тех же значениях g). В вычислениях были использованы таблицы эллип- 
тических интегралов [ $\left.{ }^{2}\right]$. Табл. 3 содержит вычисленные по формулам $(35),(36),(38)$ и (44)-(46) значения величин $C, \Theta_{0}, a, a, b$ и g(90). Отметим, что, как вытекает из формул (38) и (44),

$$
\mathrm{Q}\left(90^{\circ}\right)=C^{-1} \cos \left(\Theta_{0} / 2\right) \text {. }
$$

Степень точности эллиптического приближения характеризуется прежде всего коэффициентами $d_{k}$ (см. формулу (49)). Табл. 4 содержит их значения, вычисленные по формулам (51) и (52). Их малость наглядно иллюстрирует малость разности $r(\vartheta)-\varrho(\vartheta)$. Но можно также прямо вычислить эту разность, пользуясь табл. 2. и формулой (38). В табл. 5 приведены ее значения вместе со значениями разности $s(\vartheta)-\sigma(\vartheta)$, вычисленными по формуле (48). Из этой таблицы видно, что относительная ошибка лишь кое-где немногим превышает $10^{-3}$. В подавляющей части она гораздо меньше, особенно для $g=1,75$.

\section{Таблица 6}

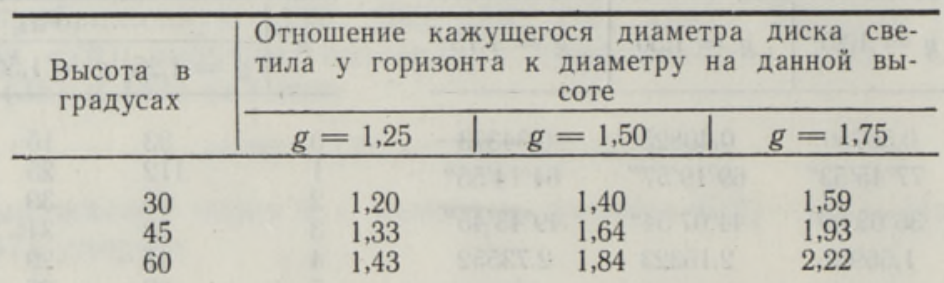

В заключение приведем еще одну таблицу. Она показывает, во сколько раз диаметр диска солнца или луны кажется у горизонта больше, чем на высоте 30,45 и $60^{\circ}$ (табл. 6). Эти числа выражаются отношениями $r\left(90^{\circ}\right) / r\left(60^{\circ}\right), r\left(90^{\circ}\right) / r\left(45^{\circ}\right)$ и $r\left(90^{\circ}\right) / r\left(30^{\circ}\right)$. Из них для средних широт наиболее репрезентативно последнее число, так как в средних широтах наибольшая высота солнца или луны близка к $60^{\circ}$. Числа даны в таблице всего с двумя знаками после запятой; при этой точности эллиптическое приближение неотличимо от точного решения.

\section{ЛИТЕРАТ У Р А}

1. Минн а р т М., Свет и цвет в природе, М., 1958.

2. С а мойлов а-Я хон тов а Н., Таблицы эллиптических интегралов, М.-Л., 1935. Тартуский государственный
университет
Поступила в редакцию 17/V 1977

\section{TAEVAVÕLVI NÄIV KUJU}

Taevavõlvi näiv lapikus on tingitud vertikaalnurkade ülehindamisest, ülehindamise algpõhjuseks on aga asjaolu, et vertikaalse eseme kõrgust hinnatakse tema kaugusega võrdseks siis, kui mōlemad pikkused (kõrgus ja kaugus) paistavad vōrdsete nurkade all. Eseme nurkkoorgust hinnatakse sel juhul vōrdseks $45^{\circ}$-ga, olgugi et ta on tegelikult väiksem. Oildjuhul kehtib valem (2), kus $\beta$ on tōeline nurkkõrgus, $\psi$ näiv nurkkõrgus, $g$ teatav isikupärane konstant $(g>1)$. See võrdub suhtega $p / h$, kus $h$ on vaatleja silma körgus maapinnast ning $p$ on maapinna selle punkti kaugus vaatieja seisupunktist, kust ta on harjunud hindama horisontaalseid kaugusi endast ettepoole. 
Neil eeldustel kehtib vertikaalringi näiva kuju määramiseks diferentsiaalvõrrand (7), kus $\theta$ on taevavõlvi mingi punkti seniitkaugus ja $r$ selle kaugus vaatlejast, algtingimusega $r(0)=1$. Vorrrandi lahend on esitatav koonduva reana (10), kus kordajad avalduvad valemitega (13) ja (15). See kõver erineb väga vähe ellipsist, mille poolteljed - horisontaalne $a$ ja vertikaalne $b-$ on määratud seostega $(35),(36)$ ja $(44)-(46)$, kus $E$ on teist liiki elliptiline integraal.

\section{P. KARD}

\section{DIE SCHEINBARE GESTALT DES HIMMELSGEWOLLBES}

Die scheinbare Abplattung des Himmelsgewölbes ist bedingt durch die Oberschätzung der Höhewinkel. Die Ursache dafür liegt im Umstande, daß die Höhe eines vertikalen Gegenstands unbewußt dem Abstande gleichgesetzt wird, wenn beide Längen (Höhe und Abstand) unter gleichen Winkeln erscheinen. Dann wird der Höhewinkel gleich $45^{\circ}$ geschätzt, obgleich er tatsächlich kleiner ist. Allgemein gilt die Formel (2), wo $\beta$ der wahre und $\psi$ der scheinbare Höhewinkel ist, während $g=p / h>1$ eine individuell bedingte Konstante ist. Hier bedeutet $h$ die Höhe des Auges des Beobachters über dem Boden und $p$ die Entfernung jenes Punktes des Bodens von Beobachters Füßen, wovon er die Längen aller horizontaler Strecken von sich in die Ferne zu schätzen gewöhnt ist. Für die scheinbare Gestalt des Vertikalkreises gilt dann die Differentialgleichung (7), wo $\vartheta$ der Zenitabstand eines Punktes des Himmelsgewölbes und $r$ dessen linearer Abstand vom Beobachter ist, wobei als Anfangsbedingung $r(0)=1 \mathrm{zu}$ nehmen ist. Die Lösung dieser Gleichung kann in der Form einer konvergenten Reihe (10) dargestellt werden, deren Koeffizienten durch die Formeln (13) und (15) festgelegt sind. Es stellt sich dann heraus, daß solchermaßen berechneter scheinbarer Vertikalkreis sehr wenig von einer Ellipse abweicht. Die Halbachsen der Ellipse - die horizontale Halbachse $a$ und die vertikale Halbachse $b-$ sind durch die Formeln $(35)$, (36) und $(44)-(46)$ ausgedrückt, wo $E$ das elliptische Integral zweiter Gattung ist. 